\title{
Effets de la destruction du gène CNTF chez la souris
}

Le facteur neurotrophique ciliaire (ainsi appelé parce que caractérisé initialement comme facteur de survie de neurones ciliaires de poulet), est une molécule exprimée après la naissance dans les cellules de Schwann myélinisées et certaines populations d'astrocytes. Sa localisation cellulaire est cytosolique. Sa fonction physiologique reste à élucider, mais sa capacité à prévenir certaines dégénérescences de neurones moteurs est établie. Ses actions trophiques in vitro sont bien connues; il permet notamment la survie des oligodendrocytes $\left(\mathrm{m} / \mathrm{s} n^{\circ} 4\right.$, vol. 9, p. 476), mais on ignore s'il possède in vivo les propriétés équivalentes. Un grand intérêt s'est attaché à son étude depuis la description de l'effet thérapeutique (non confirmé) sur la souris $\mathrm{MnD}$ (motoneural degeneration, $\mathrm{m} / \mathrm{s} n^{\circ} 2$, vol. $8, p .176$ ) et surtout, par un groupe allemand, sur la souris pmn, victime d'une atteinte motoneurale rappelant celle de la maladie de Werdnig-Hoffmann ([1] et $m / s n^{\circ} 7$, vol. $8, p$. 744). Ce même groupe (Martinsried, Allemagne) a fait faire au sujet une percée décisive en réalisant la destruction du gène CNTF chez la souris par recombinaison homologue [2].

Le gène du CNTF a été cloné en 1989 par deux groupes, l'un de Boulder, (CO, USA) [3], l'autre de Martinsried (Allemagne) [4]. La phase ouverte de lecture est de $600 \mathrm{pb}$, codant pour une protéine de 200 acides aminés, sans peptide signal. La rupture du gène a été réalisée par des méthodes désormais classiques, en insérant une cassette d'expression neo dans l'ADN génomique de CNTF. La séquence neo était suivie de plusieurs codons stop pour éviter une lecture à travers un codon de terminaison unique. On obtint des cellules ES qui furent injectées à des blastocystes de 3,5 jours. Des hétérozygotes furent accouplés et une proportion généti-

$\mathrm{m} / \mathrm{s} n^{\circ} 11$ vol. 9, novembre 93 que mendélienne fut obtenue dans la descendance. Aucune protéine ni action biologique du type CNTF ne fut observée chez les homozygotes $\mathrm{CNTF}^{-1-}$.

Comme on pouvait s'y attendre du fait de l'expression tardive normale du CNTF, aucun effet de l'inactivation ne se fit sentir pendant la période foetale. Le nombre de neurones moteurs ne subit aucune diminution à la naissance ni dans les quatre premières semaines. La situation changea par contre dès la $8^{e}$ semaine, et le nombre des neurones commença à diminuer. C'est le noyau facial qui fut choisi pour les compter, en raison de ses limites bien définies, et de l'absence de cellules d'autres types susceptibles d'interférer. A 28 semaines, le nombre de neurones moteurs était réduit de $22 \%(\mathrm{p}=0,002)$ en même temps que progressaient des changements morphologiques, perte de corps de Nissl et réactions gliales. Cliniquement les perturbations se sont montrées tardives, une légère diminution de la force musculaire apparaissant vers 28 semaines.

La destruction du gène du CNTF permet donc de constater que ce facteur n'est pas nécessaire pour le développement des neurones moteurs. Il l'est, en revanche, pour le maintien de leur intégrité postnatale. Les lésions décrites dans l'article [2] sont limitées mais s'aggraveront sans doute avec un recul plus grand, quand les animaux seront plus âgés. Restent plusieurs questions, tant théoriques que pratiques. Le CNTF, dans certains tissus, existe à un taux très élevé: il est, par exemple, 10000 fois plus concentré que le NGF dans le nerf sciatique; mais il n'est pas sécrété et reste dans le cytoplasme; il ne passe pas dans le milieu en culture cellulaire. On ne sait donc pas comment il est transféré des cellules de Schwann sur sa cible, le motoneurone. L'interruption de sa production permettra-t-elle de vérifier s'il existe in vivo un équivalent des actions observées in vitro, notamment celle de différenciation cholinergique (élévation de la choline acétyltransférase et baisse de la tyrosine hydroxylase) ? Sur le plan thérapeutique, le CNTF justifiera-t-il les espoirs qu'il a suscités ? On ne connaît pas de maladies dont soit responsable l'absence de CNTF; leur recherche impliquerait une analyse systématique de ce facteur dans les amyotrophies spinales qui n'ont pas encore été élucidées, ce qui n'a pas été fait à notre connaissance. Les effets obtenus dans le traitement des souris pmn sont vraisemblablement purement symptomatiques, mais ils ouvrent néanmoins des perspectives que médecine/sciences a déjầ soulignées $\left(n^{\circ} 7\right.$, vol. $8, p$. 744). L'emploi de méthodes relevant du génie génétique, différentes cependant du simple remplacement d'un gène non fonctionênel, permet d'espérer, à terme, une thérapeutique efficace de maladies neurodégénératives.

J.C.D.

1. Sendtner M, Schmalbruch H, Stōckli KA Carroll P, Kreutuberg GW, Thoenen H. Ciliary neurotrophic factor prevents degeneration of motor neurons in mouse mutant progressive motor neuropathy. Nature 1992 ; 358: 502-4.

2. Masu Y, Wolf E, Holtmann B, Sendtner M, Brem G, Thoenen $H$. Disruption of the CNTF gene results in motor neuron degeneration. Nature $1993 ; 365$ : 27-32.

3. Lin LFH, Mismer D, Lile JD, Armes LG, Butler III ET, Vannice JL, Collins F. Purification, cloning, and expression of ciliary neurotrophic factor (CNTF). Science 1989; 246 : 1023-5.

4. Stöckli KA, Lottspeich F, Sendtner M, Masiakowski P, Carroll P, Gōtz R, Lindholm $\mathrm{D}$, Thoenen $\mathrm{H}$. Molecular cloning, expression and regional distribution of rat ciliary neurotrophic factor. Nature 1989; 342 : 920-3. 